\title{
Lactobacillus amylophilus D14 protects tight junction from enteropathogenic bacteria damage in Caco-2 cells
}

\author{
Q. Yu, Z. Wang, and Q. Yang ${ }^{1}$ \\ College of Veterinary Medicine, Nanjing Agricultural University, Weigang 1, Nanjing, Jiangsu 210095, PR China
}

\begin{abstract}
Enterotoxigenic Escherichia coli and Salmonella Typhimurium could adhere to epithelial tissue and destroy cell junctions, leading to intestinal inflammation and diarrhea. Lactobacillus could prevent the adhesion of pathogens to host cells and protect the mucosal barrier. The objective of this study was to investigate the protective effects of Lactobacillus amylophilus D14 on Caco-2 cells against the invasion of enterotoxigenic Escherichia coli K88 and Salmonella Typhimurium SL1344. We found that with a reduction in dextran permeability and an increase in transepithelial electrical resistance, L. amylophilus D14 could ameliorate the damage to cell integrity caused by pathogens. Furthermore, $L$. amylophilus D14 reduced the expression of phosphorylated extracellular signal-regulated protein kinase and phospho-c-jun N-terminal kinase, and it decreased the secretion of IL-8. The abilities of the Lactobacillus to protect the cell junctions were then evaluated on Caco2 cells. Increased expression and amelioration distribution of tight junction proteins (zonula occludens-1, claudin-1, and E-cadherin) were observed when the cells were cocultured with pathogens and Lactobacillus simultaneously. Lactobacillus amylophilus D14 may influence the mitogen-activated protein kinase pathway to regulate the correct assembly of the tight junction and adherens junction, protecting the cell junctions and mucosal barrier damaged by enterotoxigenic $E$. coli K88 or Salmonella Typhimurium SL1344 infection.
\end{abstract}

Key words: Lactobacillus, tight junction, intestinal pathogen, Caco-2 cell

\section{INTRODUCTION}

The intestinal mucosal barrier is the largest interface separating the internal and external environments. Numberless microorganism are also living in the intestines, adhering to the epithelial tissue or invading through the mucosal barrier, or both. A critical

Received March 15, 2012.

Accepted June 21, 2012.

${ }^{1}$ Corresponding author: iamspringyqh@yahoo.com.cn component of the mucosal barrier is the cell junctions between adjacent intestinal epithelial cells, which form a semipermeable diffusion barrier. These intercellular junctions consist of the tight junction, the adherens junction, desmosomes, and the gap junction (Steed et al., 2010). The tight junction is the most apical and is responsible for controlling the permeability of the paracellular pathway. When intestinal barrier function is compromised, it can become "leaky," allowing pathogens and toxins to enter the body.

The enteropathogens may have various effects on the cell junctions and mucosal barrier. Some pathogens, such as Salmonella Typhimurium and enterotoxigenic Escherichia coli (ETEC), could adhere to the brush border of intestinal cells, damaging the structure of the tight junction or adherens junction (Roselli et al., 2003; Köhler et al., 2007). However, some probiotic bacteria exert beneficial properties in terms of maintenance or enhancement of the intestinal barrier function (Asahara et al., 2011). Probiotics are defined as live microbial food supplements that, when ingested, can survive the gastrointestinal tract (Valerio et al., 2011). The mode of action of a probiotic is complex and has not yet been fully elucidated. Some mechanisms have been reported to explain probiotic actions, such as ameliorating the intestinal permeability induced by pathogens in vitro (Klingberg et al., 2005; Sherman et al., 2005), preventing and reducing the number of pathogens adhering to intestinal cells (Yu et al., 2011), or enhancing the tight junction integrity between intestinal epithelial cells that are not weakened (Putaala et al., 2008).

Existing mechanistic studies about the tight junction and mucosal barrier have focused on the interaction between intestinal epithelial tissue and pathogens, such as Salmonella Typhimurium and Helicobacter pylori (Köhler et al., 2007; Wroblewski et al., 2009). For Lactobacillus, considerable attention has been paid to the antagonistic effects of pathogen adhesion and the protection of the mucosal barrier. The objective of this study was to investigate the protective effects of Lactobacillus amylophilus D14 against the invasion of ETEC K88 and Salmonella Typhimurium SL1344 in vitro by using human colon cancer cells (Caco-2 cells), a commonly used model of the intestinal epithelium that 
spontaneously forms a tight junction between adjacent cells. Dextran permeability; transepithelial electrical resistance (TER) assays; IL-8 secretion, measures of the expression levels of extracellular-regulated protein kinase (ERK), c-jun N-terminal kinase (JNK); and measures of tight junction proteins and fluorescent microscopy of tight junction proteins were performed to explore the effects of L. amylophilus D14 on the tight junction and mucosal barrier.

\section{MATERIALS AND METHODS}

\section{Bacterial Growth and Cell Culture}

Enterotoxigenic E. coli K88 and Salmonella Typhimurium SL1344 were grown in Luria-Bertani broth containing $1 \%$ tryptone and $0.5 \%$ yeast extract plus $1 \% \mathrm{NaCl}, \mathrm{pH}$ 7.0. After an overnight incubation at $37^{\circ} \mathrm{C}$ with vigorous shaking, the bacteria were diluted 1:100 in fresh Luria-Bertani broth and grown for $2 \mathrm{~h}$ until the mid-log phase for all experiments. Lactobacillus amylophilus D14 was selected and grown in de Man, Rogosa, Sharpe medium at $37^{\circ} \mathrm{C}$ under anaerobic conditions. After an overnight incubation, they were diluted 1:10 in fresh de Man, Rogosa, Sharpe medium and grown for $4 \mathrm{~h}$ until the mid-log phase. For each experiment, the bacteria were harvested by centrifugation at $3,000 \times g$ for $10 \mathrm{~min}$ at room temperature and then resuspended in antibiotic-free Dulbecco's modified Eagle's medium (DMEM).

The Caco-2 cells were obtained from the American Type Culture Collection (ATCC, Rockville, MD) and maintained in DMEM supplemented with $10 \%$ fetal bovine serum. The cells were incubated at $37^{\circ} \mathrm{C}$ in a humidified atmosphere of $5 \% \mathrm{CO}_{2}$ in air. The Caco-2 cells were seeded on Millicell filter inserts $(1 \mu \mathrm{m}$; Millipore Corp., Billerica, MA) at a seeding density of $10^{4}$ cells $/ \mathrm{cm}^{2}$. Cells reached confluence in 7 to $8 \mathrm{~d}$. The cell culture medium was changed to fresh medium without antibiotics before treatment of the cells with bacteria.

\section{Dextran Permeability as Measurements of Changes in the Barrier Function of Polarized Epithelial Cell Monolayers}

Polarized Caco-2 cells were grown on Millicell filter inserts and cultured until the TER reached a minimum of $1,000 \Omega / \mathrm{cm}^{2}$. Before bacterial infection, the cells were washed and placed in antibiotic-free medium. The cells were then treated with $1 \mathrm{~mL}$ of medium containing ETEC K88 or Salmonella Typhimurium SL1344 $\left(10^{8}\right.$ cfu/well), or L. amylophilus D14 $\left(10^{9} \mathrm{cfu} /\right.$ well $)$ either alone or simultaneously for $2 \mathrm{~h}$. The movement of macromolecules across polarized epithelial cell monolayers was assayed by using a macromolecular conjugate probe, fluorescein-isothiocyanate dextran, of molecular masses of $70 \mathrm{kDa}$ (FD 70S; Sigma, St. Louis, MO). Briefly, $0.2 \mathrm{~mL}$ of conjugated dextran suspended in DMEM was added to the apical compartment of the Millicell filter inserts, and $0.4 \mathrm{~mL}$ of DMEM alone was added to the basolateral compartment. After incubation for $2 \mathrm{~h}$ at $37^{\circ} \mathrm{C}$, samples $(0.1 \mathrm{~mL})$ from the apical and basolateral compartment were placed into a 96 -well plate (Corning, Tewksbury, MA) and analyzed to determine their fluorescent intensity using a Bio-Tek fluorescence spectrophotometer (excitation wavelength 495 $\mathrm{nm}$, emission wavelength $525 \mathrm{~nm}$ ). The permeability of these probes was expressed as the percentage ratio of the concentration in the basolateral chamber versus that in the apical chamber.

\section{Measurement of TER and IL-8 Assay}

Lactobacillus amylophilus D14 $\left(10^{9} \mathrm{cfu} /\right.$ well $)$ or enteropathogenic bacteria (ETEC K88 or Salmonella Typhimurium SL1344, $10^{8} \mathrm{cfu} /$ well) diluted in DMEM without antibiotic were added to the apical compartment of Millicell filter inserts (Millipore Corp.) either alone or simultaneously for $2 \mathrm{~h}$. Control Caco-2 cells were cultured only with DMEM. The TER assay was performed on the Caco-2 cell monolayers at $0,1,2,3$, 4,6 , and $12 \mathrm{~h}$ by measuring the TER using a MillicellERS-II Volt Ohm meter (Millipore Corp.). Meanwhile, after incubation for $6 \mathrm{~h}\left(37^{\circ} \mathrm{C} ; 5 \% \mathrm{CO}_{2}\right)$, the basolateral medium was collected and used for IL-8 assay. The IL- 8 concentration in the basolateral medium was determined with the human IL-8 ELISA kit (Boster, Wuhan, China).

\section{Analysis of Zonula Occludens-1, Claudin-1, and E-Cadherin Distribution by Fluorescence Microscopy}

The Caco-2 cell monolayers were treated as described previously. When the Caco-2 cells reached confluence on the Millicell filter inserts (Millipore Corp.), L. amylophilus D14 ( $10^{9} \mathrm{cfu} /$ well) or enteropathogenic bacteria (ETEC K88 or Salmonella Typhimurium SL1344, $10^{8} \mathrm{cfu} /$ well) diluted in DMEM without antibiotic were added to the apical compartment of the Millicell filter inserts either alone or simultaneously for $2 \mathrm{~h}$. Control Caco-2 cells were cultured only with DMEM. The immunofluorescence staining protocol was adapted from the protocol (Zareie et al., 2005). Briefly, confluent Caco-2 cell monolayers were rinsed in PBS, followed by fixation and permeabilization in $5 \%$ formaldehyde $\left(20^{\circ} \mathrm{C}\right)$ for $15 \mathrm{~min}$. The Caco- 2 cells were then incubated in $5 \%$ (vol/vol) normal goat serum in PBS for 
$1 \mathrm{~h}$ at room temperature and then incubated with primary antibodies against zonula occludens-1 (ZO-1), claudin-1, or E-cadherin (Zymed, San Francisco, CA) at $37^{\circ} \mathrm{C}$ overnight. After unbound primary antibodies were rinsed away with PBS, cells or tissues were incubated with secondary cyanine 3- (Cy3) conjugated goat anti-rabbit IgG (1:200 dilution; Zymed) or fluorescein isothiocyanate-conjugated goat anti-mouse IgG (1:500 dilution; Boster) for $1 \mathrm{~h}$ at room temperature. The Caco-2 cells were thoroughly rinsed with PBS and examined with a fluorescence microscope (BX5, Olympus, Tokyo, Japan).

\section{Analysis of ERK and JNK Expression on Caco-2 Cells by Western Blotting}

The Caco-2 cell monolayers were treated as described previously and then collected immediately and snap-frozen in liquid nitrogen. In preparation for SDS-PAGE, cells were thawed to $4^{\circ} \mathrm{C}$ and homogenized in chilled radioimmunoprecipitation assay buffer, including protease and phosphatase inhibitors. After centrifugation at $10,000 \times g$ for $10 \mathrm{~min}$ at $4^{\circ} \mathrm{C}$, the supernatant was recovered and assayed for protein concentrations, determined by the Bradford method. Equal amounts of total protein were separated on $10 \%$ SDS-polyacrylamide gels and then transferred to the polyvinylidene fluoride membrane. After blocking overnight in Tris-buffered saline containing $0.05 \%$ Tween and $5 \%$ dry powdered milk, membranes were incubated at $4{ }^{\circ} \mathrm{C}$ in primary antibodies (antibodies specific for phosphorylated forms of ERK1/2, JNK1/2/3, total ERK1/2, total JNK1/2/3). After 3 washes with Tris-buffered saline containing $0.05 \%$ Tween, the membranes were incubated for $1 \mathrm{~h}$ with horseradish peroxidase-conjugated secondary antibody. The membranes were then developed for visualization of protein by adding an enhanced chemiluminescence reagent (Pierce, Rockford, IL). Autoradiograms were scanned and analyzed with Quantity One (Bio-Rad, Hercules, CA) to quantify band densities. The mean density of the phosphorylated ERK or JNK band was divided by the mean density of the corresponding total ERK or JNK band to yield a normalized band density value.

\section{Analysis of ZO-1, Claudin-1, and E-Cadherin Expression by Western Blotting}

The Caco-2 cell monolayers were collected immediately snap-frozen in liquid nitrogen. Western blot intensity measurements for whole-cell proteins were determined from the ratio of the integrated intensity of the cell junction protein (claudin-1, ZO-1, or E-cadherin) bands to the integrated intensity of the $\beta$-actin band in the same sample.

\section{Statistical Analysis}

The results were expressed as means $\pm \mathrm{SE}$ of the means. Statistical differences between multiple groups were calculated using ANOVA.

\section{RESULTS}

\section{L. amylophilus D14 Reduced the Permeability of FD 70 S on Caco-2 Cells Infected with Salmonella Typhimurium SL1344}

The results showed that L. amylophilus D14 treatment alone did not influence the diffusion of the probe (as measured by the signal intensity for basal medium samples) from apical to basolateral Millicell compartments (relative integrated intensity compared with untreated monolayers; Figure 1A). Compared with the ETEC K88 infection (1.57 \pm 0.08$)$, Salmonella Typhimurium SL1344 induced more severe damage to the integrity of the cells and caused a large increase in permeability of FD $70 \mathrm{~S}(3.97 \pm 0.07, P<0.01)$. When Caco-2 cells were simultaneously treated with L. amylophilus D14, they exhibited a marked decrease $(P<0.01)$ in the permeability to FD $70 \mathrm{~S}$ compared with the cells infected with ETEC K88 or Salmonella Typhimurium SL1344 alone.

\section{L. amylophilus D14 Attenuated ETEC K88- or Salmonella Typhimurium SL1344-Induced Decreases in TER}

As observed, treatment of polarized epithelial cells with L. amylophilus D14 alone did not influence the TER of polarized Caco- 2 cell monolayers and performed the balance within $12 \mathrm{~h}$ (Figure 1B). However, ETEC K88 or Salmonella Typhimurium SL1344 infection resulted in a significant reduction in the TER of Caco-2 cells $(13.2 \pm 3.7 \%$ of the TER of cell monolayers at $0 \mathrm{~h}$ or $11.2 \pm 0.2 \%$ at $12 \mathrm{~h}, P<0.01)$. Lactobacillus amylophilus D14 attenuated the reduction of TER of cells infected with ETEC K88 or Salmonella Typhimurium SL1344 $(P<0.01)$.

\section{L. amylophilus D14 Counteracted the ETEC K88- or Salmonella Typhimurium SL1344-Induced Dysregulation of IL-8 Expression}

The secretion of IL-8 was evaluated when Caco2 cells were cocultured with ETEC K88, Salmonella 
Typhimurium SL1344, or L. amylophilus D14 alone or simultaneously (Figure 2). Compared with the untreated cells, treatment with L. amylophilus D14 did not modify the expression of IL-8 $(160.1 \pm 11.0 \mathrm{pg} /$ $\mathrm{mL})$. Compared with the cells infected with ETEC K88 $(209.6 \pm 19.4 \mathrm{pg} / \mathrm{mL})$, treatment with L. amylophilus D14 significantly abrogated the increase in IL-8 (115.3 $\pm 2.8 \mathrm{pg} / \mathrm{mL}, P<0.01)$. Similar results were detected with Salmonella Typhimurium infection $(305.8 \pm 9.5$ $\mathrm{pg} / \mathrm{mL})$. Lactobacillus amylophilus D14 also had the ability to reduce the secretion of IL- 8 significantly $(196.9 \pm 10.8 \mathrm{pg} / \mathrm{mL}, P<0.01)$.

\section{Expression of ERK and JNK on Caco-2 Cells Cocultured with $L$. amylophilus D14}

We next investigated the activation of ERK and JNK in polarized Caco-2 cells treated with L. amylophilus D14 alone or with pathogens simultaneously. The results demonstrated that L. amylophilus D14 did not alter the expression of the ERK phosphorylation level (Figure $3)$. In contrast, significantly increased expression of pERK was detected in Caco-2 cells infected with ETEC K88 or Salmonella Typhimurium SL1344 $(P<0.01)$. However, L. amylophilus D14 was able to significantly inhibit this increase in phosphorylation level of ERK $(P$ $<0.01$ ). The increase in phosphorylation level of JNK with ETEC K88 infection could also be significantly ameliorated by treatment with L. amylophilus D14 ( $P$ $<0.01$; Figure 3).

\section{Influence of L. amylophilus D14 on the Redistribution and Expression of Tight Junction Proteins}

The effect of L. amylophilus D14 and pathogens on the distribution of ZO-1, claudin-1, and E-cadherin was evaluated. Lactobacillus amylophilus D14 did not alter the distribution of tight junction proteins, which were present at the apical intercellular borders in a belt-like manner, encircling the cells and delineating the cellular borders (Figure 4). The fluorescence was discontinuous and was dispersed into the cytoplasm in cells infected with ETEC K88 or Salmonella Typhimurium SL1344. However, L. amylophilus D14 was able to ameliorate the abnormal distribution of junction proteins, whereas the green or red spots were increased compared with ETEC or SL1344 infection.

Western blotting of epithelial tissue whole-cell protein extracts (Figure 5) showed a significant reduction in ZO-1, claudin-1, and E-cadherin expression in cells infected with ETEC K88 or Salmonella Typhimurium SL1344 $(P<0.01)$. However, L. amylophilus D14 did not influence the expression of ZO-1, claudin-1, and E-

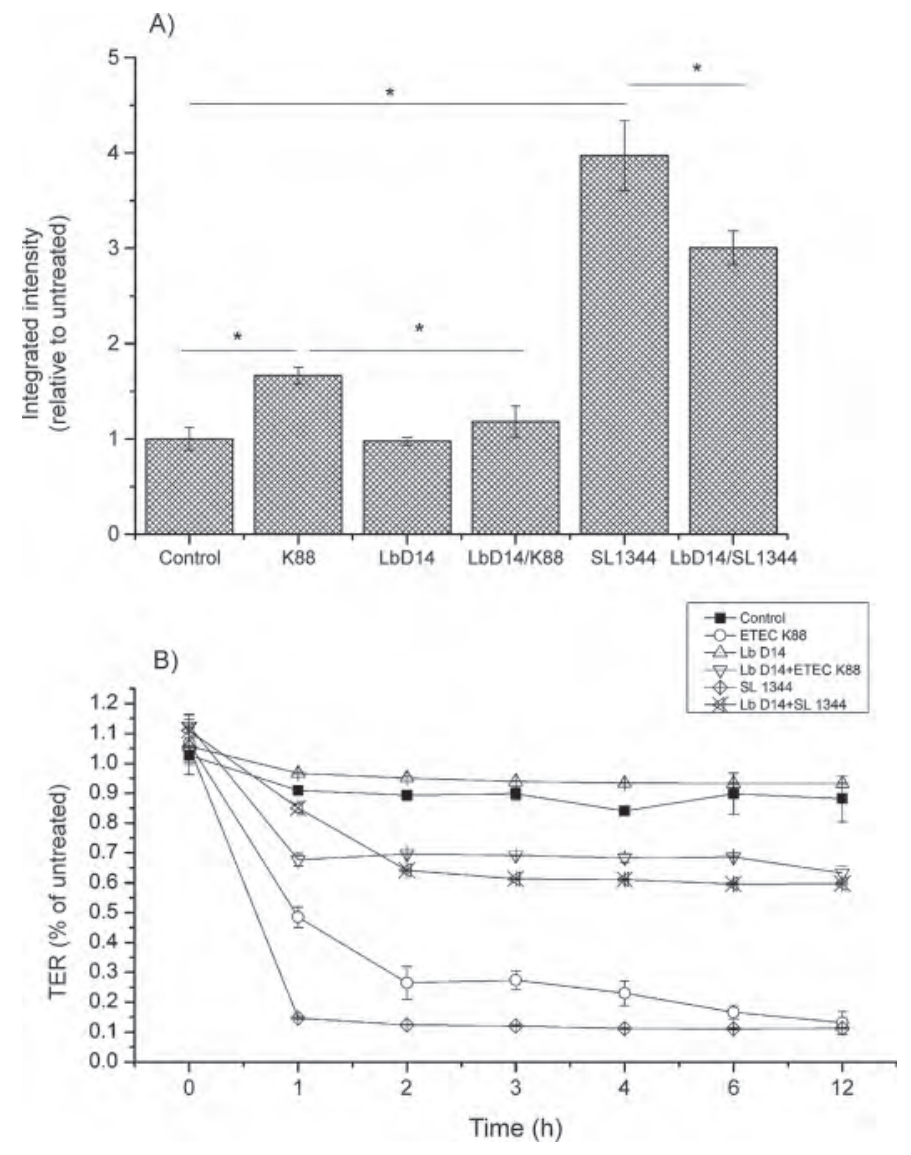

Figure 1. Effects of Lactobacillus amylophilus D14 (Lb D14) on dextran permeability (A) and transepithelial electrical resistance (TER; B). Integrated intensities of dextran diffused into the basolateral compartments of Millicells (Millipore Corp., Billerica, MA) containing Caco-2 cells $2 \mathrm{~h}$ after addition to the apical compartment. Polarized cells were treated with L. amylophilus D14 or pathogens [enterotoxigenic Escherichia coli (ETEC) K88 or Salmonella Typhimurium SL1344] simultaneously. ${ }^{*} P<0.01$, as determined by ANOVA. Data represent the means $\pm \mathrm{SE}$ from 4 independent experiments.

cadherin. Furthermore, when treated with Lactobacillus and enteropathogenic bacteria concurrently, Caco-2 cells maintained higher levels of ZO-1, claudin-1, and E-cadherin expression than did cells infected with the pathogen alone (Figure 5).

\section{DISCUSSION}

The integrity of the mucosal barrier is important to defend against the invasion of pathogenic bacteria. Cellular confluence and mucosal integrity have often been verified by measurements of dextran permeability and transepithelial electrical resistance (Cruz et al., 1994; Lambert et al., 2005). The increased permeability of FD 70S in Caco-2 cells, which were infected with ETEC K88 or Salmonella Typhimurium SL1344 in $2 \mathrm{~h}$, indicated that the mucosal integrity was damaged and 


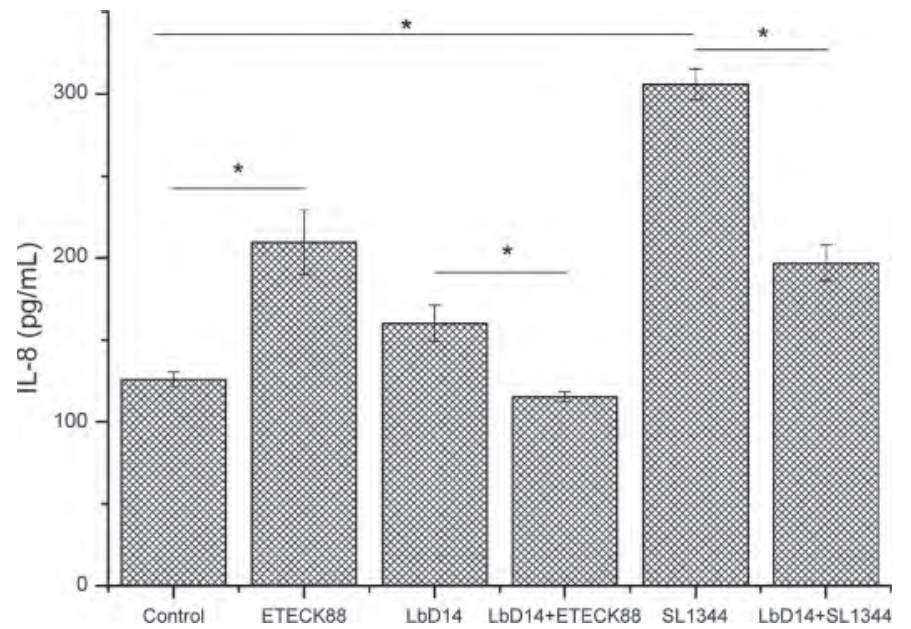

Figure 2. Effect of Lactobacillus amylophilus D14 (Lb D14) on IL-8 production. Interleukin- 8 production was measured by ELISA after cells were treated with L. amylophilus D14 or pathogens [enterotoxigenic Escherichia coli (ETEC) or Salmonella Typhimurium SL1344] simultaneously for $6 \mathrm{~h}$. ${ }^{*} P<0.01$, as determined by ANOVA. Data represent the means $\pm \mathrm{SE}$ from 4 independent experiments.

the paracellular pathway was out of control. However, this phenomenon was ameliorated when Caco-2 cells were cocultured with L. amylophilus D14. Furthermore, results of the TER assay were consistent with the permeability of dextran. Enterotoxigenic E. coli K88 or Salmonella Typhimurium SL1344 infection caused a rapid decrease in TER in $1 \mathrm{~h}$. However, L. amylophilus D14 was able to delay the decline in TER value and keep the TER value at a stable level.

Interleukin-8 was able to be produced by epithelial cells and to induce the accumulation of neutrophil granulocytes, leading to inflammation in vivo. The secretion of IL-8 was detected to verify whether L. amylophilus D14 could prevent the inflammation caused by ETEC K88 and Salmonella Typhimurium SL1344. The Caco-2 cells infected with ETEC K88 and Salmonella Typhimurium SL1344 stimulated high expression of IL8. This was consistent with previous reports (Shaughnessy et al., 2009; Devriendt et al., 2010). When Caco-2 cells were cocultured with L. amylophilus D14 for $6 \mathrm{~h}$, the secretion of IL- 8 was significantly reduced $(P<$ 0.01) compared with cells infected with ETEC K88 or Salmonella Typhimurium SL1344 alone. The results demonstrated that L. amylophilus D14 could restrain inflammation.

We also detected the distribution and expression of ZO-1, claudin-1, and E-cadherin to observe the effects of L. amylophilus D14 on cell junction proteins. Zonula
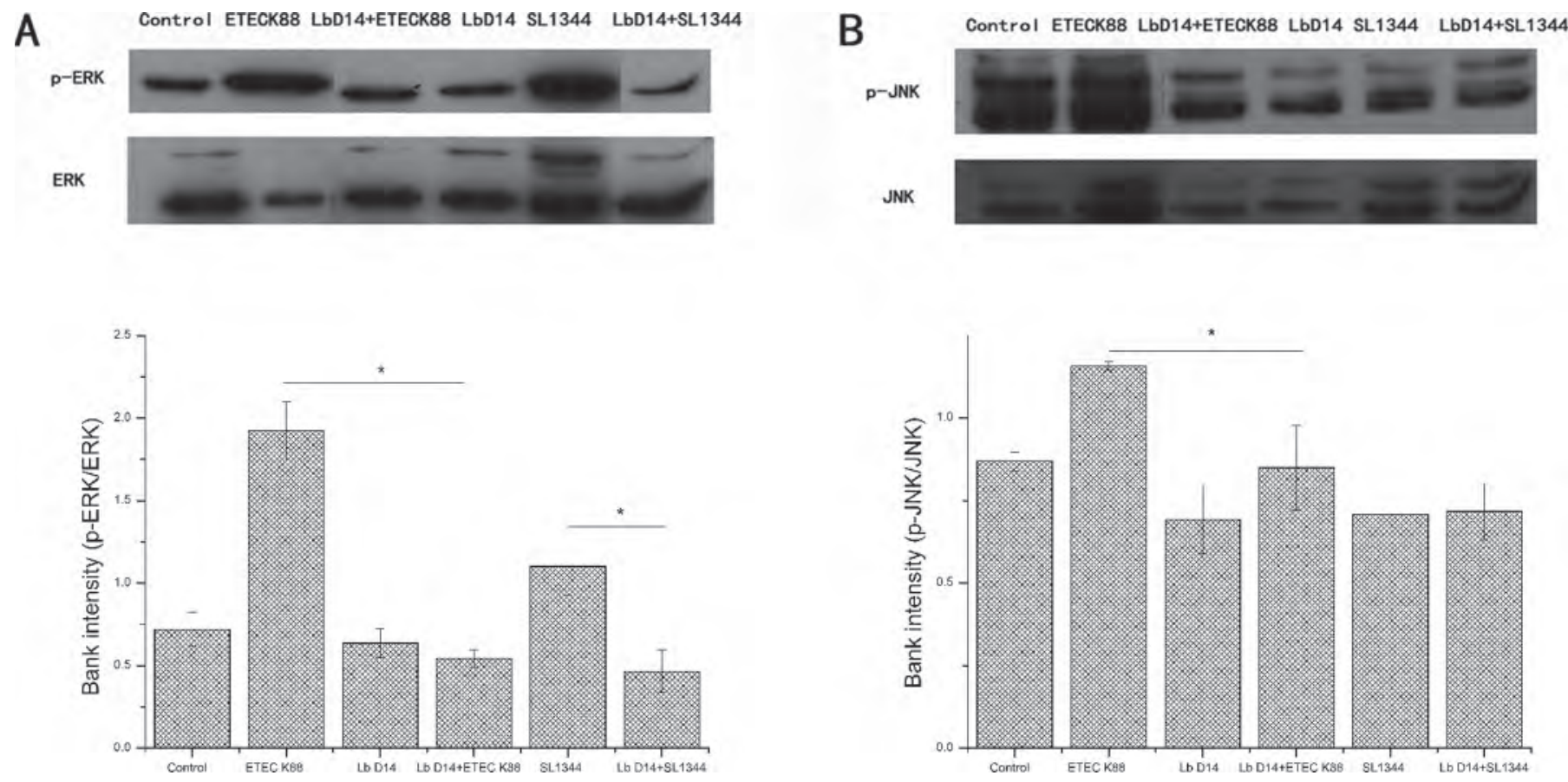

Figure 3. Lactobacillus amylophilus D14 (Lb D14) inhibited the overexpression of phosphorylated extracellular-regulated protein kinase (pERK) and c-jun N-terminal kinase (p-JNK) caused by enterotoxigenic Escherichia coli (ETEC) or Salmonella Typhimurium on Caco-2 cells. Caco-2 cells were untreated (control), infected with ETEC K88 or Salmonella Typhimurium SL1344 alone, or cocultured with L. amylophilus D14 simultaneously for $2 \mathrm{~h}$. The figure shows a Western blot of immunoprecipitated ERK and JNK levels, representative of 4 independent assays, and the densitometric values of p-ERK and p-JNK normalized to total ERK and JNK. Values are means \pm SE, ${ }^{*} P<0.01$, as determined by ANOVA. 


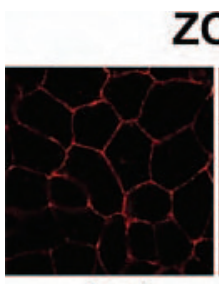

Control

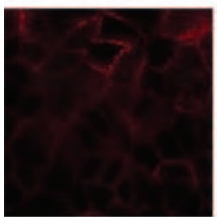

K 88

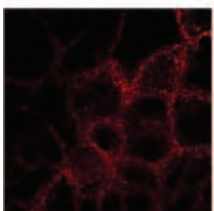

SL1344
ZO-1

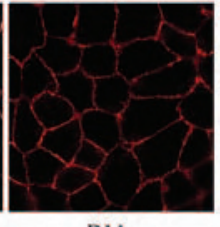

D14

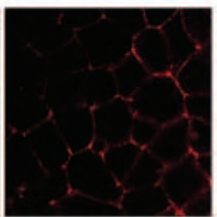

K88+D14

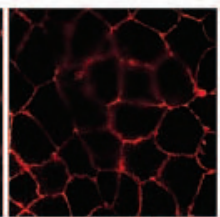

SL1344+D14
Claudin-1

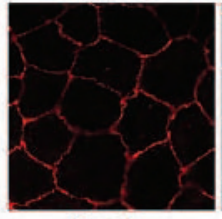

Control

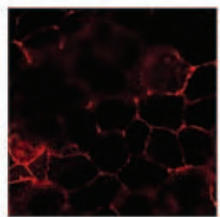

K88

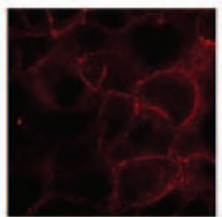

SL1344

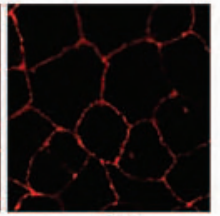

D14

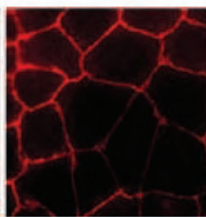

K88+D14

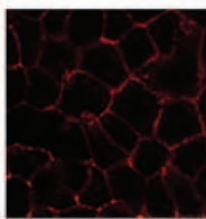

SL1344+D14
E-cadherin

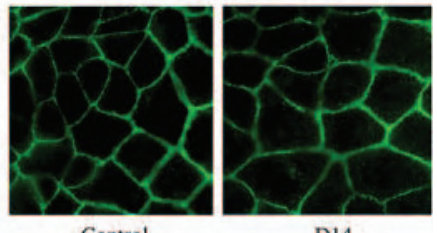

Control

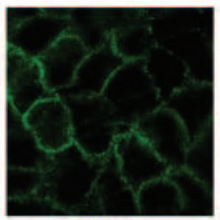

K 88

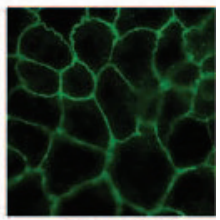

K88+D14

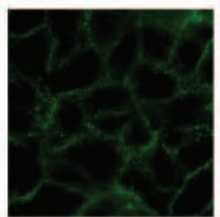

SL1344

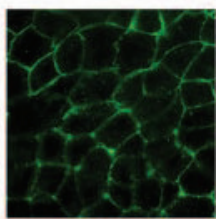

SL1344+D14

Figure 4. Enterotoxigenic Escherichia coli (ETEC) K88 or Salmonella Typhimurium SL1344 infection caused redistribution of zonula occludens-1 (ZO-1; red), claudin-1 (red), and E-cadherin (green) in Caco-2 cell monolayers 2 h postinfection. The ETEC K88 or Salmonella Typhimurium SL1344 could alter the distribution of ZO-1, claudin-1, and E-cadherin. However, when Caco-2 cells were cocultured with Lactobacillus amylophilus D14 (D14) and pathogenic bacteria, L. amylophilus D14 prevented the changes in claudin-1 and E-cadherin redistribution caused by ETEC K88 or Salmonella Typhimurium SL1344. These data are representative of 4 independent assays. Color version available in the online PDF.

occludens-1 and claudin-1 are important proteins of the tight junction complex, whereas E-cadherin constitutes the adherens junction and regulates assembly of the tight junction complex (Troxell et al., 2000). The distribution of ZO-1, claudin-1, and E-cadherin was altered in Caco-2 cells infected with ETEC K88 or Salmonella Typhimurium SL1344. The fluorescence was discontinuous along the cell border and dispersed into the cytoplasm, implying that the proteins diffused into the cytoplasm from the cell membrane. The phenomenon was similar to a Helicobacter pylori infection (Wroblewski et al., 2009). These changes correlate with the increase in paracellular permeability of FD 70S and IL-8 secretion, as well as reduction of the TER value. To support the microscopy observations, we also used Western blotting techniques to determine the expression levels of cell junction proteins. The results demonstrated that the expression levels of claudin-1, ZO-1, and E-cadherin were decreased significantly with ETEC or Salmonella Typhimurium SL1344 infection, compared with the control group. The decreased expression and unbalanced distribution of the proteins were improved in Caco-2 cells cocultured with L. amylophilus D14 for $2 \mathrm{~h}$, indicating the protective effects of Lactobacillus on cell junctions and mucosal integrity.
Tight junction assembly and function can be modulated by several signaling molecules, whereas the mitogen-activated protein kinase (MAPK) pathways are important in maintaining the integrity of the junctions. Extracellular-regulated protein kinase and JNK are 2 key components of MAPK (Cargnello and Roux, 2011). Previous reports demonstrated that after inhibition of the MAPK pathway, occludin, claudin-1, and ZO-1 were recruited to the cell membrane, the tight junction was assembled, and E-cadherin protein expression was induced in Madin-Darby canine kidney cells (Chen et al., 2000). Activation of the ERK1/2 MAPK pathway induces tight junction disruption in human corneal epithelial cells (Wang et al., 2004). Similar results were also observed in the present study. Expression levels of the phosphorylation of ERK and JNK were significantly increased in cells infected with ETEC or Salmonella Typhimurium SL1344, whereas expression levels of claudin-1, ZO-1, and E-cadherin were reduced. However, L. amylophilus D14 reduced the overexpression of p-ERK and JNK to normal levels and increase the expression levels of junction proteins, leading to the correct assembly of the tight junction.

In conclusion, L. amylophilus D14 may influence the MAPK pathway to regulate the correct assembly of the 


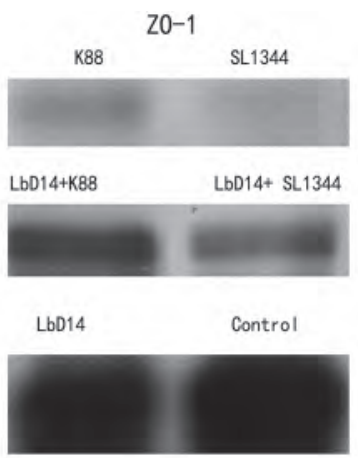

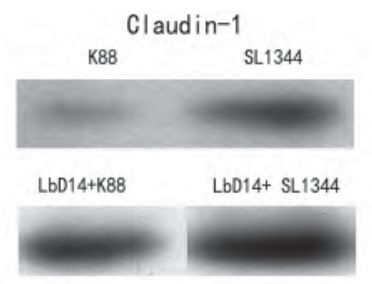

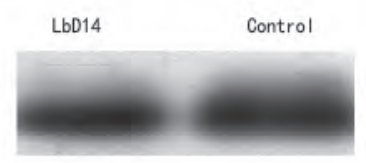

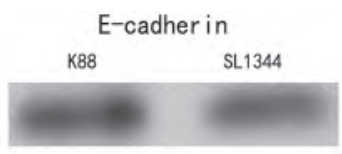
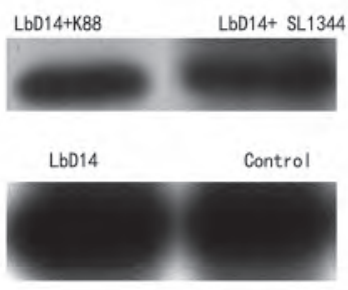
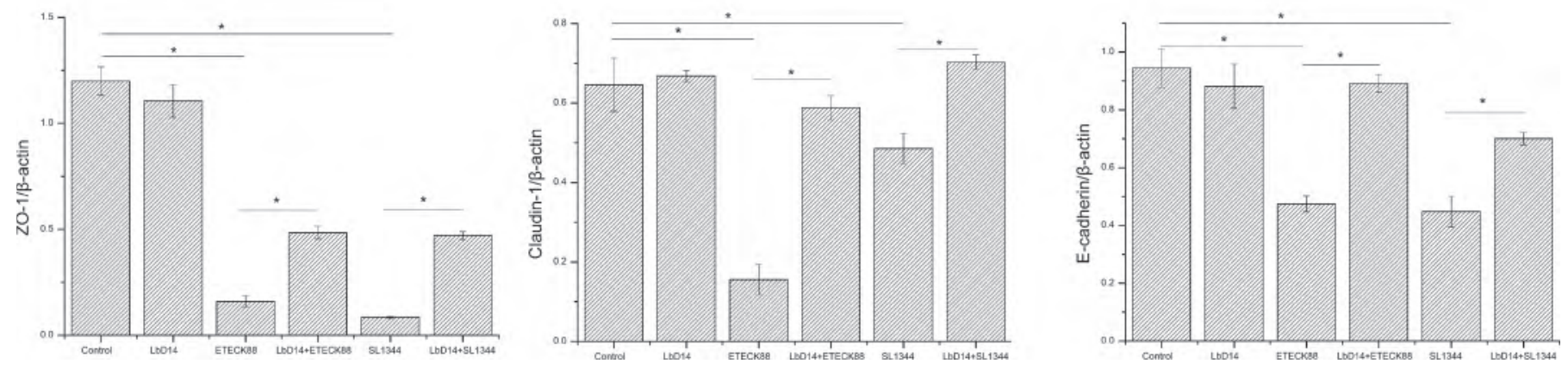

Figure 5. Lactobacillus amylophilus D14 (Lb D14) inhibited the zonula occludens-1 (ZO-1), claudin-1, and E-cadherin reduction caused by enterotoxigenic Escherichia coli (ETEC) K88 or Salmonella Typhimurium SL1344 on Caco-2 cells. Caco-2 cells were untreated (control), infected with ETEC or Salmonella Typhimurium alone, or cocultured with Lactobacillus strains simultaneously for $2 \mathrm{~h}$. The figure shows a Western blot of immunoprecipitated ZO-1, claudin-1, and E-cadherin levels, representative of 4 independent assays, and the densitometric values of ZO-1, claudin-1, and E-cadherin normalized to $\beta$-actin. Values are means $\pm \mathrm{SE},{ }^{*} P<0.01$, as determined by ANOVA.

tight junction and the adherens junction, protecting the cell junctions damaged by ETEC or Salmonella Typhimurium SL1344 infection. Finally, the effects were reflected by an improvement in the TER value and the reduction of FD 70S permeability and IL-8 secretion. These results provide new insights into the protective activity of L. amylophilus D14 against ETEC or Salmonella Typhimurium SL1344 infection.

\section{ACKNOWLEDGMENTS}

This work was supported by the research fund for the doctoral program of higher education of China (No. 20100097120007), a Jiangsu Province Natural Science Foundation grant (No. BK2011645) and Nanjing Agricultural University Young Teacher Fund (KJ09014) and Priority Academic Program Development (PAPD). No potential conflicts (financial, professional, or personal) are relevant to the manuscript.

\section{REFERENCES}

Asahara, T., K. Shimizu, T. Takada, S. Kado, N. Yuki, M. Morotomi, R. Tanaka, and K. Nomoto. 2011. Protective effect of Lactobacillus casei strain Shirota against lethal infection with multi-drug resis- tant Salmonella enterica serovar Typhimurium DT104 in mice. J. Appl. Microbiol. 110:163-173.

Cargnello, M., and P. P. Roux. 2011. Activation and function of the MAPKs and their substrates, the MAPK-activated protein kinases. Microbiol. Mol. Biol. Rev. 75:50-83.

Chen, Y., Q. Lu, E. E. Schneeberger, and D. A. Goodenough. 2000. Restoration of tight junction structure and barrier function by down-regulation of the mitogen-activated protein kinase pathway in ras-transformed Madin-Darby canine kidney cells. Mol. Biol. Cell 11:849-862.

Cruz, N., L. Qi, X. Alvarez, R. D. Berg, and E. A. Deitch. 1994. The Caco-2 cell monolayer system as an in vitro model for studying bacterial-enterocyte interactions and bacterial translocation. J. Burn Care Rehabil. 15:207-212.

Devriendt, B., E. Stuyven, F. Verdonck, B. M. Goddeeris, and E. Cox. 2010. Enterotoxigenic Escherichia coli (K88) induce proinflammatory responses in porcine intestinal epithelial cells. Dev. Comp. Immunol. 34:1175-1182.

Klingberg, T. D., M. H. Pedersen, A. Cencic, and B. B. Budde. 2005. Application of measurements of transepithelial electrical resistance of intestinal epithelial cell monolayers to evaluate probiotic activity. Appl. Environ. Microbiol. 71:7528-7530.

Köhler, H., T. Sakaguchi, B. P. Hurley, B. A. Kase, H. C. Reinecker, and B. A. McCormick. 2007. Salmonella enterica serovar Typhimurium regulates intercellular junction proteins and facilitates transepithelial neutrophil and bacterial passage. Am. J. Physiol. Gastrointest. Liver Physiol. 293:G178-G187.

Lambert, D., C. A. O'Neill, and P. J. Padfield. 2005. Depletion of Caco-2 cell cholesterol disrupts barrier function by altering the detergent solubility and distribution of specific tight-junction proteins. Biochem. J. 387:553-560.

Putaala, H., T. Salusjarvi, M. Nordstrom, M. Saarinen, A. C. Ouwehand, E. Bech Hansen, and N. Rautonen. 2008. Effect of four pro- 
biotic strains and Escherichia coli O157:H7 on tight junction integrity and cyclo-oxygenase expression. Res. Microbiol. 159:692-698.

Roselli, M., A. Finamore, I. Garaguso, M. S. Britti, and E. Mengheri. 2003. Zinc oxide protects cultured enterocytes from the damage induced by Escherichia coli. J. Nutr. 133:4077-4082.

Shaughnessy, R. G., K. G. Meade, S. Cahalane, B. Allan, C. Reiman, J. J. Callanan, and C. O'Farrelly. 2009. Innate immune gene expression differentiates the early avian intestinal response between Salmonella and Campylobacter. Vet. Immunol. Immunopathol. 132:191-198.

Sherman, P. M., K. C. Johnson-Henry, H. P. Yeung, P. S. Ngo, J. Goulet, and T. A. Tompkins. 2005. Probiotics reduce enterohemorrhagic Escherichia coli O157:H7- and enteropathogenic E. coli O127:H6-induced changes in polarized T84 epithelial cell monolayers by reducing bacterial adhesion and cytoskeletal rearrangements. Infect. Immun. 73:5183-5188.

Steed, E., M. S. Balda, and K. Matter. 2010. Dynamics and functions of tight junctions. Trends Cell Biol. 20:142-149.

Troxell, M. L., S. Gopalakrishnan, J. McCormack, B. A. Poteat, J. Pennington, S. M. Garringer, E. E. Schneeberger, W. J. Nelson, and J. A. Marrs. 2000. Inhibiting cadherin function by dominant mutant E-cadherin expression increases the extent of tight junction assembly. J. Cell Sci. 113:985-996.
Valerio, F., S. de Candia, S. L. Lonigro, F. Russo, G. Riezzo, A. Orlando, P. De Bellis, A. Sisto, and P. Lavermicocca. 2011. Role of the probiotic strain Lactobacillus paracasei LMGP22043 carried by artichokes in influencing faecal bacteria and biochemical parameters in human subjects. J. Appl. Microbiol. 111:155-164.

Wang, Y., J. Zhang, X. J. Yi, and F. S. Yu. 2004. Activation of ERK1/2 MAP kinase pathway induces tight junction disruption in human corneal epithelial cells. Exp. Eye Res. 78:125-136.

Wroblewski, L. E., L. Shen, S. Ogden, J. Romero-Gallo, L. A. Lapierre, D. A. Israel, J. R. Turner, and R. M. Peek Jr. 2009. Helicobacter pylori dysregulation of gastric epithelial tight junctions by ureasemediated myosin II activation. Gastroenterology 136:236-246.

Yu, Q., Z. Wang, and Q. Yang. 2011. Ability of Lactobacillus to inhibit enteric pathogenic bacteria adhesion on Caco-2 cells. World J. Microbiol. Biotechnol. 27:881-886.

Zareie, M., J. Riff, K. Donato, D. M. McKay, M. H. Perdue, J. D. Soderholm, M. Karmali, M. B. Cohen, J. Hawkins, and P. M. Sherman. 2005. Novel effects of the prototype translocating Escherichio coli, strain C25 on intestinal epithelial structure and barrier function. Cell. Microbiol. 7:1782-1797. 